\title{
Candida colonization in the nursery
}

\author{
Paolo Manzoni, ${ }^{1}$ Michael Mostert, ${ }^{2}$ E. Jacqz-Aigrain, ${ }^{3}$ Mauro Stronati, ${ }^{4}$ Daniele Farina $^{1}$
}

In this issue, Pinhat et al. ${ }^{1}$ report on a cohort study in which very low birth weight (VLBW) preterm neonates underwent structured microbiological surveillance, and describe the profile and characteristics of fungal colonization in neonatal intensive care units (NICUs) and the related risk factors in these premature infants.

Colonization by fungal species (mainly Candida) is a common occurrence in preterm neonates and poses serious management and diagnostic problems. ${ }^{2}$

Similar to all other types of patients admitted to an intensive care unit (ICU), preterm neonates have a greater than usual risk of getting colonized while in the ICU, and this is a matter of concern due to the high odds of progression towards systemic dissemination that have been reported in such patients. ${ }^{3-5}$

The estimated frequency of Candida colonization in premature newborn patients during their stay in the NICU ranges between 10 and $60 \%$ of all VLBW infants. ${ }^{1}$ Whereas the risk of fungal infection is decreased with the use of fresh maternal milk, ${ }^{6}$ the latter does not protect the neonate from the risk of fungal gut colonization. ${ }^{7}$

There is a wide range of variability in the reported frequencies of fungal colonization in NICUs due to several factors. Inconsistencies in surveillance culture policies make it difficult to compare the incidence rates of different settings and institutions: as a result, NICUs performing weekly (or semiweekly) cultures for each patient will claim higher incidence rates of colonization than those that do not have such a policy. Moreover, it is known that in preterm neonates colonization originates from both acquired (horizontal transmission) and endogenous source, and also from colonized mothers (vertical transmission). These two different modalities show some overlap over time, making it difficult to ascertain both the origin and frequency of colonization in neonatal settings.

In addition, the definition of colonization is not properly established: the most used one is "colonization is defined as the isolation of Candida spp. from at least one surveillance culture during the NICU stay," but it is clear that this definition is somewhat controversial and far from definitive.

Among all fungal organisms, preterm infants get colonized mostly (if not only) by the various Candida spp. The mechanisms of colonization involve mainly adhesion of the fungus to the host. The different steps of adhesion include the formation of superficial abscesses, invasion of deep tissues with formation of microabscesses in organs, and systemic dissemination. All these steps depend on the number of fungal organisms, their virulence, and the characteristics of the host response. Adhesion is indeed the key factor for colonization. A number of factors regulate this process, including glucose (that enhances the expression of fungal CR3 receptors), steroids (that enhance the expression of fungal genes), and genetics (since there are different levels of expression of the adhesin INT-1 gene).

1. MD. Neonatology and Neonatal Intensive Care Unit (NICU), Sant'Anna Hospital, Torino, Italy.

2. MD. Department of Paediatrics, University of Torino, Italy.

3. MD, PhD. Department of Paediatric Pharmacology and Pharmacogenetics, Clinical Investigation Center Inserm, Hospital Robert Debrè, Paris, France.

4. MD. Neonatology and NICU, IRCCS Policlinico San Matteo, Pavia, Italy.

No conflicts of interest declared concerning the publication of this editorial.

Suggested citation: Manzoni P, Mostert M, Jacqz-Aigrain E, Stronati M, Farina D. Candida colonization in the nursery. J Pediatr (Rio J). 2012;88(3):187-90. http://dx.doi.org/10.2223/JPED.2201 
Also, the humidity of the environment, as well as the type of fungal species ( $C$. albicans adhere more than $C$. tropicalis or C. glabrata) and their different ability to form biofilms on venous catheters, are crucial variables to consider in this process. ${ }^{8}$

In premature infants in the NICU, the most common sites of colonization are the gastrointestinal tract (in the study by Pinhat et al., ${ }^{1} 89 \%$ of the cases were reported in the rectal mucosa), the urinary tract, the skin, the central venous catheter (CVC), the endotracheal tube and any other mechanical device.

Overcrowding in the NICU, low nurse-to-patient ratio and poor hygiene practices are associated with the onset of fungal colonization. Other independent risk factors for colonization include vaginal delivery, as pointed out by Pinhat et al., ${ }^{1}$ skin disruption, disturbances of the enteric microbiota, use of mechanical devices kept in place overtime, and use of certain medications (e.g. antibiotics, steroids) that enhance Candida spp. growth. In particular, the duration of broad-spectrum antibiotic therapy is a potent factor that independently promotes fungal growth as measured through the colonization index in nonneutropenic ICU patients hospitalized for more than 7 days. ${ }^{9}$

The premature neonate is an interesting model to assess the timing of onset of fungal colonization in an ICU. Most ICU patients are exposed to fungal organisms, a number of them actually become colonized, and only a minority develops systemic dissemination originating from peripheral colonization. Reports from patients admitted to ICUs other than NICUs (e.g. surgical ICUs) show that the risk for colonization is higher in the presence of peripheral and central intravenous catheters, bladder catheters, mechanical ventilation, and lack of enteral nutrition. ${ }^{10}$ This also applies to preterm neonates in NICUs.

The timing of colonization steps has been elucidated through a number of studies that could point out a peak of colonization in VLBW infants in the NICU between the 14th and 28th day of life, ${ }^{11}$ and that the overall risk for colonization changes over time during the NICU stay. It has been demonstrated that the period during which patients have a CVC in place, are under broad-spectrum antibiotics, and receive total parenteral nutrition (TPN) poses a higher risk for colonization. ${ }^{12}$ In the study by Pinhat et al., ${ }^{1}$ the average hospitalization time was 30.5 days ( \pm 20.27 ), and the onset of the colonization occurred, in average, at 11.13 days $( \pm 8.82)$. This is in accordance with other reports describing that Candida colonization is inversely proportional to gestational age and occurs on the skin (first NICU week) and gastrointestinal tract (second NICU week) prior to the respiratory tract (third NICU week). In this view, C. albicans is more likely than C. parapsilosis to colonize multiple sites. ${ }^{13}$

Monitoring Candida colonization in NICU patients is crucial because colonization is a strong and reliable marker for potential invasive infection, and hence the importance of early detection to answer the identification question of which colonized patients will ultimately progress to infection.

In all ICU settings, Candida spp. colonization proved to be an independent risk factor for invasive Candida infection (ICI) and candidemia. ${ }^{14}$ The literature shows that among all ICU patients, those who are colonized have the highest odds of suffering from invasive fungal disease after progression from colonization to systemic dissemination. Moreover, differently colonized ICU patients have different odds of developing invasive disease depending on a number of factors that are inherent to the type of patient, the microorganism involved, and the characteristics of colonization (e.g. sites and intensity). ${ }^{15}$

Patient-related risk factors for progression from colonization to infection (Table 1 ) include the severity of the underlying clinical conditions (expressed through the various APACHE, CRIB, APGAR scores), concomitant surgery (mainly in the gastrointestinal tract), concomitant antifungal prophylaxis, presence of skin or mucosal breakdown, disruption of the intestinal barrier, use of concomitant medications, presence of mechanical devices, or the need for invasive procedures.

Organism-related risk factors for progression from colonization to infection (Table 2) include the anatomic site (type of site), the intensity of colonization (number of sites involved and number of colonies), the timing of this process, and the species involved.

It has been calculated that $5-30 \%$ of all colonized preterm neonates will develop ICI during their stay in the NICU. ${ }^{16} \mathrm{~A}$ retrospective study involving more than 200 VLBW colonized infants showed that the only significant risk factors for progression to ICIs were CVC colonization $(p<0.001)$ and multiple-site colonization (at least three sites) $(p=0.001)$. A lower birth weight was inversely correlated with progression, but this was only of borderline significance $(p=0.06)$. It is worth noting that fluconazole prophylaxis was the only significant protective factor $(p=0.03) .{ }^{16}$

The anatomic site of colonization is a potential predictor of invasiveness. In the literature, isolation of Candida spp. from the urinary tract ${ }^{17}$ and from endotracheal samples $^{18}$ has been associated with the highest odds of progression to ICI.

Likewise, intensity of colonization, usually expressed as the number of non-contiguous sites concomitantly involved, is a predictor of invasiveness. ${ }^{19}$ In preterm neonates, having more than three sites concomitantly colonized translates into a 6.1 -fold increase in the odds of progression, ${ }^{15}$ and having more than four sites translates into up to 12.1-fold increase in the odds of progression towards systemic dissemination. ${ }^{20}$

Suspected or documented Candida colonization poses important management issues for the NICU, and joint 
Table 1 - Patient-related risk factors for progression to invasive Candida infections in colonized patients in the ICU

\begin{tabular}{lllll}
\hline $\begin{array}{l}\text { Immune } \\
\text { disorders }\end{array}$ & $\begin{array}{c}\text { Medications } \\
\text { that facilitate } \\
\text { fungal growth }\end{array}$ & $\begin{array}{c}\text { Medications } \\
\text { that suppress } \\
\text { immune function }\end{array}$ & Diseases & Conditions \\
\hline - Neutropenia & - Cephalosporins & - H2-blockers & - Focal bowel perforation & - Severity \\
- Immunosuppression & - Carbapenems & $\begin{array}{l}\text { (and likely proton } \\
\text { pump inhibitors) }\end{array}$ & - Complicated GI diseases & - CVC in place \\
& & - Steroids & - Hyperglycemia or diabetes & - Intubation \\
\hline
\end{tabular}

$\mathrm{CVC}=$ central venous catheter; $\mathrm{GI}=$ gastrointestinal; $\mathrm{ICU}=$ intensive care unit.

Table 2 - Organism-specific risk factors for invasive Candida infections in colonized patients in the ICU

\begin{tabular}{clll}
\hline $\begin{array}{c}\text { Adherence } \\
\text { properties }\end{array}$ & $\begin{array}{c}\text { Virulence properties } \\
\text { (ability to disseminate) }\end{array}$ & $\begin{array}{c}\text { Ability of a single } \\
\text { subspecies to colonize } \\
\text { multiple sites }\end{array}$ & $\begin{array}{c}\text { Patterns } \\
\text { of colonization }\end{array}$ \\
\hline - Genetic variability & - C. albicans +++ & - C. albicans +++ & - Cutaneous candidiasis \\
& - C. glabrata ++ & - C. parapsilosis + & - Multiple-site colonization \\
& - C. parapsilosis + & - CVC colonization & - Inoculum intensity \\
\hline
\end{tabular}

$\mathrm{CVC}=$ central venous catheter; $\mathrm{ICU}=$ intensive care unit.

actions, in collaboration with the microbiology laboratory, are strongly recommended due to the importance of culture and species identification.

Antifungal prophylaxis with fluconazole has proven effective in preventing colonization, 12,21 but it has an uncertain effect on the rates of progression towards invasion. ${ }^{21}$ A recent European survey performed by the TINN Consortium ${ }^{22}$ pointed out that, at the present stage, more than half of the European NICUs are adopting prophylactic fluconazole strategies, and that indications for use of fluconazole in neonates and infants are related to the prevention and treatment of Candida infections.

Prophylaxis with bovine lactoferrin has emerged as a promising alternative to prevent fungal infections in the NICU, although it does not reduce the rates of enteric colonization by Candida spp. ${ }^{23}$

Surveillance cultures in the NICU are very helpful if we know how to manage them properly. These policies should be solely intended as a tool to help describe and monitor fungal ecology in the ICU, especially when adopting the routine use of fluconazole, which might, in theory, potentiate the emergence of resistant Candida spp. The detection of a fungus isolated from sites other than blood, CVC, cerebrospinal fluid, peritoneal fluid, and urine (collected using sterile procedures) should never prompt the implementation of systemic therapy with antifungal drugs, because the treatment of colonization itself is not appropriate.

In selected cases, in accordance with similar policies adopted in other ICU settings, preemptive therapy with systemic antifungal drugs based on the "colonization status" data can be considered. This might be a feasible option in neonates not protected by prophylactic fluconazole and featuring an intense and rapidly progressing colonization in sites with typically high odds of systemic dissemination. 


\section{References}

1. Pinhat EC, Borba MG, Ferreira ML, Ferreira MA, Fernandes RM, Nicolau SK, et al. Fungal colonization in newborn babies of very low birth weight: a cohort study. J Pediatr (Rio J). 2012;88:211-6.

2. Kaufman D, Fairchild KD. Clinical microbiology of bacterial and fungal sepsis in very-low-birth-weight infants. Clin Microbiol Rev. 2004; 17:638-80.

3. Safdar A, Armstrong D. Prospective evaluation of Candida species colonization in hospitalized cancer patients: impact on short-term survival in recipients of marrow transplantation and patients with hematological malignancies. Bone Marrow Transplant. 2002;30:931-5.

4. Safdar N, Maki DG. The commonality of risk factors for nosocomial colonization and infection with antimicrobialresistant Staphylococcus aureus, enterococcus, gram-negative bacilli, Clostridium difficile, and Candida. Ann Intern Med. 2002;136:834-44.

5. León C, Ruiz-Santana S, Saavedra P, Almirante B, Nolla-Salas J, Alvarez-Lerma F, et al. A bedside scoring system ("Candida score") for early antifungal treatment in nonneutropenic critically ill patients with Candida colonization. Crit Care Med. 2006;34:730-7.

6. Schanler RJ, Lau C, Hurst NM, Smith EO. Randomized trial of donor human milk versus preterm formula as substitutes for mothers' own milk in the feeding of extremely premature infants. Pediatrics. 2005;116:400-6.

7. Manzoni $P$, Mostert M, Stronati M. Lactoferrin for prevention of neonatal infections. Curr Opin Infect Dis. 2011;24:177-82.

8. Bendel CM. Colonization and epithelial adhesion in the pathogenesis of neonatal candidiasis. Semin Perinatol. 2003;27:357-64.

9. Charles PE, Dalle F, Aube H, Doise JM, Quenot JP, Aho LS, et al. Candida spp. colonization significance in critically ill medical patients: a prospective study. Intensive Care Med. 2005;31:393-400.

10. McKinnon PS, Goff DA, Kern JW, Devlin JW, Barletta JF, Sierawski $\mathrm{S}$, et al. Temporal assessment of Candida risk factors in the surgical intensive care unit. Arch Surg. 2001;136:1401-8.

11. Kicklighter SD, Springer SC, Cox T, Hulsey TC, Turner RB. Fluconazole for prophylaxis against candidal rectal colonization in the very low birth weight infant. Pediatrics. 2001;107:293-8.

12. Kaufman D, Boyle R, Hazen KC, Patrie JT, Robinson M, Donowitz LG. Fluconazole prophylaxis against fungal colonization and infection in preterm infants. N Engl J Med. 2001;345:1660-6.

13. Kaufman DA, Gurka MJ, Hazen KC, Boyle R, Robinson M, Grossman LB. Patterns of fungal colonization in preterm infants weighing less than 1000 grams at birth. Pediatr Infect Dis J. 2006;25:733-7.
14. Manzoni P, Benjamin DK, Hope W, Rizzollo S, Del Sordo P, Stronati $M$, et al. The management of Candida infections in preterm neonates and the role of micafungin. J Matern Fetal Neonatal Med. 2011;24:24-7.

15. Manzoni P, Farina D, Galletto P, Leonessa M, Priolo C, Arisio R, et al. Type and number of sites colonized by fungi and risk of progression to invasive fungal infection in preterm neonates in neonatal intensive care unit. J Perinat Med. 2007;35:220-6.

16. Manzoni $P$, Farina D, Leonessa $M, d \backslash$ 'Oulx EA, Galletto $P$, Mostert $M$, et al. Risk factors for progression to invasive fungal infection in preterm neonates with fungal colonization. Pediatrics. 2006;118:2359-64.

17. Rowen JL, Rench MA, Kozinetz CA, Adams JM Jr, Baker CJ. Endotracheal colonization with Candida enhances risk of systemic candidiasis in very low birth weight neonates. J Pediatr. 1994; 124:789-94.

18. Wynn JL, Tan S, Gantz MG, Das A, Goldberg RN, Adams-Chapman $\mathrm{I}$, et al. Outcomes following candiduria in extremely low birth weight infants. Clin Infect Dis. 2012;54:331-9.

19. Mahieu LM, Van Gasse N, Wildemeersch D, Jansens H, Ieven M. Number of sites of perinatal Candida colonization and neutropenia are associated with nosocomial candidemia in the neonatal intensive care unit patient. Pediatr Crit Care Med. 2010;11:240-5.

20. El-Masry FA, Neal TJ, Subhedar NV. Risk factors for invasive fungal infection in neonates. Acta Paediatr. 2002;91:198-202.

21. Manzoni P, Stolfi I, Pugni L, Decembrino L, Magnani C, Vetrano G, et al. A multicenter, randomized trial of prophylactic fluconazole in preterm neonates. N Engl J Med. 2007;356:2483-95.

22. Kaguelidou F, Pandolfini C, Manzoni P, Choonara I, Bonati M, JacqzAigrain E. European survey on the use of prophylactic fluconazole in neonatal intensive care units. Eur J Pediatr. 2012;171:439-45.

23. Manzoni P, Stolfi I, Messner H, Cattani S, Laforgia N, Romeo MG, et al. Bovine lactoferrin prevents invasive fungal infections in very low birth weight infants: a randomized controlled trial. Pediatrics. 2012;129:116-23.

Correspondence:

Dr. Paolo Manzoni, MD

Neonatology and NICU, S. Anna Hospital

Azienda Ospedaliera Regina Margherita, S. Anna, C.so Spezia 60

10126 - Torino - Italy

Tel. : +39 (011) 313.4304, +39 (011) 313.4305,

+39 (011) 313.4308

Fax: +39 (011) 313.4888

E-mail: paolomanzoni@hotmail.com 